\title{
New Trends in the Field of Propylene Based Polymers
}

\author{
P. GALli, ${ }^{*}$ F. Milani, ${ }^{* *}$ and T. SimONAZZI ${ }^{* * *}$ \\ *MONTEDISON/DUTRAL S.p.A., Milano, Italy \\ ${ }^{* *}$ MONTEDISON/DUTRAL S.p.A., Ferrara, Italy \\ ***HIMONT ITALIA S.p.A., Ferrara, Italy
}

(Received August 20, 1984)

\begin{abstract}
This paper deals with the high degree of control of the intimate structure of polymers obtained with Ziegler-Natta $(Z-N)$ catalysts. In the field of the poly( $\alpha$-olefin)s, there are $\mathrm{Z}-\mathrm{N}$ catalysts able to produce high density polyethylene, isotactic polypropylene (PP) or ethylenepropylene (EP)-copolymers including the EP-elastomers. The heterophasic phenomena and in particular the toughening of PP with amorphous ethylene-propylene copolymers have been studied. By terpolymerization of propylene, ethylene and other monomer such as 1-butene, terpolymers with very interesting performance are produced, thanks to the addition of 1-butene to ethylene-propylene copolymers. A decrease in $T_{\mathrm{g}} \delta$ takes place in raw polymers, while polyethylene crystaliinity is found at higher ethylene content than is the corresponding ethylene-propylene copolymers. An increase in tensile strength and a decrease in permanent deformation is noticed, both at environment and low temperature for the vulcanized products compared to the traditional ethylene-propylene copolymers. By the use of the above catalysts, copolymers and saturated terpolymers free from low volatile substance may be produced. Production of these new copolymers and saturated terpolymers can be carried out by highly simplified processes, mainly consisting of a polymerization and a monomer flashing step only, and the well known advantages have already been achieved with the new processes for the production of poly $(\alpha$-olefin)s.

KEY WORDS Propylene Copolymers / Toughening Effects / Terpolymers Ethylene-Propylene-Butene-1 / Production Processes / Block Copolymer / Random Copolymers / Ethylene-Propylene Elastomers /
\end{abstract}

Few catalyst families are as versatile as ZieglerNatta (Z-N) catalysts and, to our knowledge, none offered such a high degree of control on the intimate structure of polymers. Olefin polymerization only should suffice to make the case. Z-N catalysts are capable of synthesizing the whole range of products from its high crystallinity extremes, represented by isotactic polypropylene (PP) and high density polyethylene (HDPE) to the totally amorphous ones, as EPR elastomers.

There is practically nothing missing among intermediate products either. Propylene and ethylene random copolymers, "block copolymers" or more appropriately called heterophasic copolymers, and others can be produced by these catalysts. The above mentioned polymers are in most cases obtained with specialized catalyst families often very different from each other. For example, the current manufacturing processes usually make use of a number of Ti-salt based catalysts for crystalline polymers and various $\mathrm{V}$-salt based catalysts for amorphous elastomeric polymers. Today, the coming of the new generation of Ti-salt based high-yield catalysts dissolved such an "ancestral memory." This catalyst family, which is supported by $\mathrm{MgCl}_{2}$ in an active form and has recently been more and more successfully commercialized, offers new capabilities of controlling the produced polymers with respect to both the intimate and granular morphology and the molecular structure.

\section{HIGH YIELD ZIEGLER-NATTA CATALYSTS}

Montedison has been working on and using highyield $\mathrm{MgCl}_{2}$ catalysts since 1968 . This choice allowed us to pursue the four major objectives of an ideal catalyst: very high activity, selectivity, control 
of structure and polymer size. Our research involved the study of the structure and behaviour of $\mathrm{MgCl}_{2}$ and the compounds resulting from its interaction with the other elements of the catalyst system. It is well known how $\mathrm{MgCl}_{2}$ based catalysts show a very high activity, in comparison with conventional ones. This is attributed mainly to a more efficient use of the transition metal atoms leading to a higher number of active centers and causing a tremendous increase in the efficiency of titanium ${ }^{1,2}$ and some increase in the chain growth rate constant. $\mathrm{MgCl}_{2}$ offers two additional interesting possibilities:

- Due to its acid nature, $\mathrm{MgCl}_{2}$ reacts with a large number of Lewis bases. Some of them allow a clear improvement of both the activity and selectivity of the catalyst. ${ }^{3}$ By shifting the complex equilibria in our heterogeneous systems consisting of $\mathrm{MgCl}_{2}$ supported titanium compounds, Lewis bases and aluminium alkyls, we can control the mechanisms involved in the initiation, growth and termination of polymerization, which determine the crystallinity and the molecular weight distribution (MWD).

- Starting from $\mathrm{MgCl}_{2}$ we can form a large number of different compounds covering a wide range of physical characteristics. By selecting the most suitable characteristic, one of the most important being a low melting point, we can adjust the shape, size, and physical structure of the catalyst. This catalyst is finely tuned for synthesizing a granular polymer of the desired shape and compactness, which meets the ten basic requirements considered indispensable to advantageously replace pellets. ${ }^{4}$ This leads to significant savings in energy and time during processing and higher versatility.

The new generation of catalysts made it possible to rationalize the manufacture of polyolefins and to simplify the process to two stages only; reaction and separation of the unconverted monomer from the polymer. ${ }^{4}$ Due to the exciting performance and versatility, these catalysts have been gaining more and more ground over all other types. They are definitely better than the low-yield technologically awkward titanium catalysts. They are also to be preferred to chromium catalysts which, besides not being able to yield a wide enough range of high quality polymers, have been questioned because of the potential health hazards involved. It is a proven fact that most new and technologically advanced processes for the synthesis of poly $(\alpha$-olefin)s and all those involving high yield catalysts for the production of polypropylene employ titanium salts supported by $\mathrm{MgCl}_{2}$ in an active form.

Let us now briefly consider two examples of polymers manufactured on an industrial scale:

\section{Polypropylene Heterophasic Copolymers}

It is well known that polypropylene performs extremely well at high temperatures, but its brittleness at relatively low temperatures represents a drawback that spoils to a large extent its otherwise excellent qualities. In order to eliminate this problem, two main methods have been independently studied and followed since the beginning. One was copolymerizing polypropylene with ethylene (or butene); the other has been mechanical blending of the homopolymer with elastomeric and/or polyethylenic matrices. Considering that the toughening of polypropylene must be achieved without penalizing its main qualities and without making use of expensive techniques, one can state that neither of the two methods has led to satisfactory overall results. In fact, mechanical blending of "discrete" phases into the homopolymeric matrix presents both technical and economical limitations. On one hand it is hard to homogenize mechanically discrete phase with a high molecular weight (MW) or with different MW. On the other, investment and maintenance costs are much higher than those necessary for a synthesis process. Unfortunately, producing high quality polymers by synthesis alone was a very complex and burdensome process which had to be carried out in separate steps with a negative impact on costs and quality of the final product. The less than sufficient know-how in the field had, and still has, such a negative impact that the term "block copolymers" was coined and widely accepted to designate this class of products! As a matter of fact, knowledge in the field, especially our control on catalyst systems, was hazy enough to prevent us for a long time from identifying one objective in structural terms and then from achieving it with simple and economical polymerization processes. On the other hand, research on the toughening of PP has never been as wide as in the case of polystyrene and acrylonitrile-butadiene-styrene (ABS) resin. Some authors $s^{5-8}$ did study the effect of the structural and morphological effects 
of the components on the continuity of the phases and on the quality of the product, but considered only products obtained through mechanical blending. Our Centro Ricerche Giulio Natta attempted successfully to solve the problem with a study aimed at finding a coherent model of a toughened PP copolymer which could be used to synthesize the product on an industrial scale. The solution was a highly crystalline polypropylene matrix and a second phase, very different but very thoroughly dispersed represented by a totally amorphous ethylene-propylene bipolymer, capable of confering elasticity within the widest possible range of temperatures. Figure 1 illustates the coherent model of a toughened PP copolymer. If the composition of the two phases is kept constant, the highest impact resistance is reached when the rubbery particles with a diameter of 1 micron are evenly distributed and well bonded throughout the crystalline matrix..$^{9-11}$ The role of the rubbery phase is to proliferate crazes, the formation of which renders possible the absorption of the shock energy at impact and during the propagation of the fracture. ${ }^{12}$ This confers to the product a higher impact resistance with respect to random copolymers. Of course, for the same composition, the higher is the amount of rubbery copolymers dispersed, the higher the impact resistance of the PP and the lower the stiffness. The balance point between these two fundamental characteristics will be set according to the application. The best impact resistance is achieved for bipolymers when the two monomers are in a 1:1 molar ratio, and the distribution is completely random, i.e., the rubber is totally amorphous. This is confirmed by dynamical-mechanical measurements with Rheovibron of various bipolymers. Figure 2 shows the $T_{\mathrm{g}} \delta$ curve as a function of temperature. Here the phase-difference angle between impact and response (damping) which represents a viscoelastic property of the material. The larger the damping, the greater the predominace of the viscous effect on the elastic one therefore the greater the capability of the system to absorb the shock energy. This clearly explains why bipolymers containing $40-50 \%$ ethylene by weight have the highest value of mechanical damping, naturally provided that the distribution of the monomer is totally random. The effect of the crystallinity of the bipolymer can be assessed either by considering the hehaviour of products containing the same amount

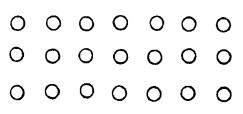

Figure 1. Coherent model of a toughened polypropylene with amorphous ethylene-propylene bipolymer. The latter phase is illustrated by circles.

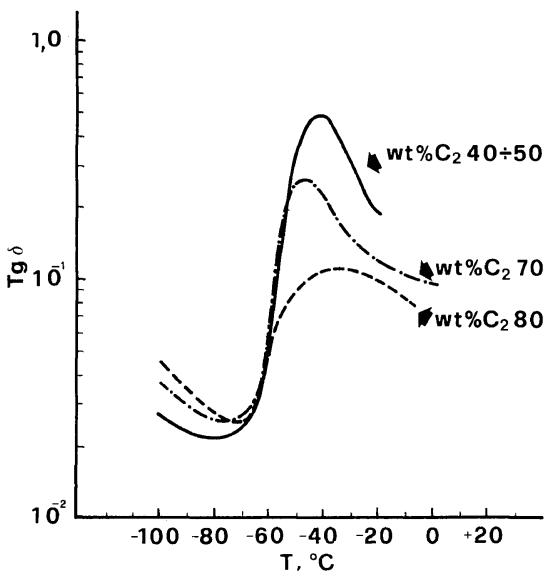

Figure 2. Effect of ethylene content on the $T_{\mathrm{g}} \delta$ versus temperature curve of amorphous ethylene-propylene bipolymers.
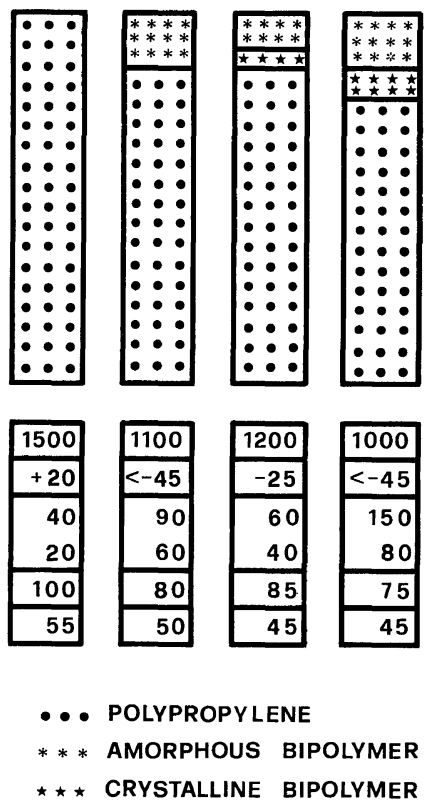

Figure 3. Effect of the crystallinity of bipolymer phase on the mechanical properties of polypropylene. 
of bipolymer with an increasing value of crystallinity due to ethylene blocking, or by keeping constant the amount of elastomer and increasing the crystalline bipolymer at the expense of the total crystalline homopolymeric phase (Figure 3 ). The following should be noted:

- In the first case with the increase in the crystalline bipolymer at the expense of the amorphous one (the third column from the left), there is a slight increase of the flexural modulus and a simultaneous decrease of the impact resistance.

-In the second case (the fourth colurnn), the increase of the bipolymeric crystalline fraction reduces the flexural modulus, but leads to no significant improvement of the impact characteristics.

In the same way, it can be demonstrated that bipolymers either containing more than $50 \%$ of their weight in $\mathrm{C}_{3}$ or with a polypropylene crystallinity show an unsatisfactory behaviour and performance. Once the model to reach was clear enough, getting there with one simple, unbroken polymerization process meant devicing and studying a particularly versatile catalyst system. It was really asking a lot from catalysis! In fact, the model requires the simultaneous presence of maximally crystalline homopolymeric phase and a totally amorphous copolymeric phase which has to be well dispersed in the first one in spherical particles with a diameter of about 1 micron. All this must be the job of only one catalytic system and is a real challenge to the adaptability and versatility of catalysts. Even though it almost seems too good to be true, there are such catalysts. The new high yield catalysts based on $\mathrm{Ti}$ salts supported on active $\mathrm{MgCl}_{2}$, are able to meet the challenge if the properly modified Lewis bases are employed. In other words, it is possible to obtain a very highly crystalline PP phase with isotactic index (I.I.) values reaching $99 \%$ and an elastomeric phase perfectly and randomly distributed in the former approaching very closely the ideal model (Figure 4).

\section{Polypropylene Statistical Copolymers}

The increasing demand of low temperature thermoweldable films has pushed research into studying the possibility of lowering the melting temperature of PP by binding smail amounts of comonomers to the main chain. Total tacticity is so reduced as well as the average length of stereoregular segments. The degree to which the melting temperature is lowered is a function of the amount of comonomer introduced and of its distribution along the main chain. It is possible to show theoretically ${ }^{13}$ that results are optimized in the case of catalytic systems with the reactivity ratio product $r_{1} \times r_{2}$ tending to zero (Figures 5 and 6). Experiments confirm the theory. Figures $7-9$ report respectively the lowering of crystallinity, of random factor (i.e., the ratio between isolated and total ethylene sequences) and of melting temperature, as functions of the amount of ethylene comonomer.

Industrial manufacturing implies a number of other factors, such as the amount of amorphous fraction soluble in the reaction medium and extractable during the centrifuging phase (Figure 10), the apparent density (Figure 11), the flowability (Figure 12) of powder polymer and other factors have to be considered. Spectacular results can be obtained if two different monomers are introduced rather than one. Terpolymerization of propylene with ethylene and butene in particular is capable of reducing drastically the melting temperature, much more than is bipolymerization with the same amount of modifier (Figure 13). This phenomenon has been explained ${ }^{14}$ by postulating the preferential formation, during terpolymerization, of the crystalline $\gamma$-triclinic-form of polypropylene, rather than the usual $\alpha$-monoclinic-form (Figure 14). The $\gamma$-form appears when short isotactic chains are present and therefore it melts at a lower temperature (Figure 15). By increasing the amount of comonomers progressively, the total $\mathrm{C}_{3}$ crystallinity is reduced proportionally and amorphous polymers are produced simultaneously in the raw polymerization material.

Finally, amorphous, elastomeric PP copolymers aroused a great deal of interest among poly- $\alpha$ olefinic amorphous products. So it became an obvious approach trying to extend the advantages inherent to the utilization of high yield catalysts to the production of EP elastomers. This research is today the most challenging one for the new frontier of Z-N catalysts. The trend is that of combining the use of particular high yield supported catalysts with the suspension process, so as to obtain elastomeric ethylene-propylene copolymers equal in chemical purity to the best polymers produced by very expensive depuration processes which decrease residual 


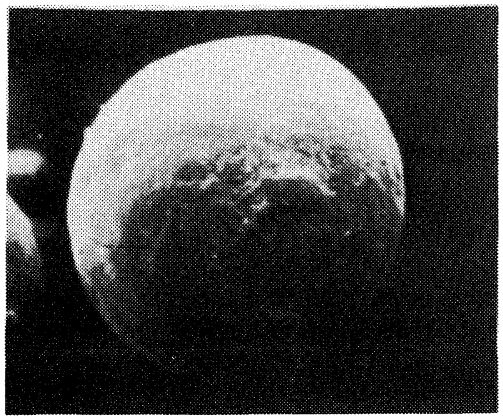

PP Copolymer-scan $(\times 60)$

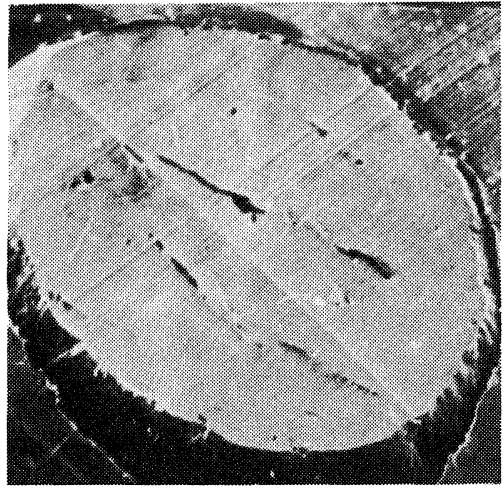

Cutted surface-scan $(\times 60)$

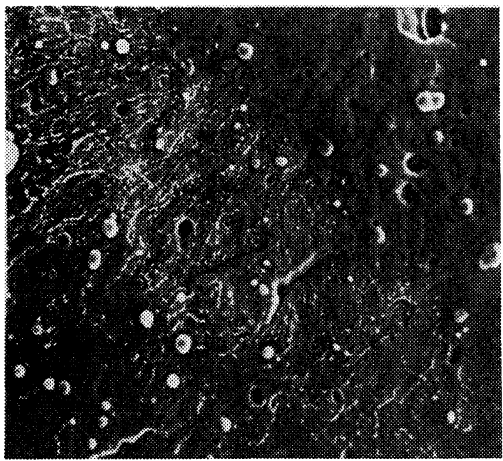

Francture surface-scan $(\times 3000)$ e

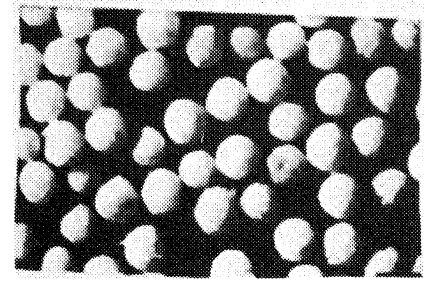

Idem-opt. microscopy $(\times 4)$ b

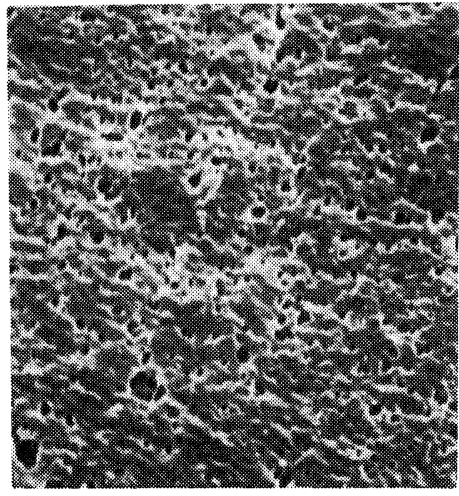

Etched surface-scan $(\times 3000)$ d

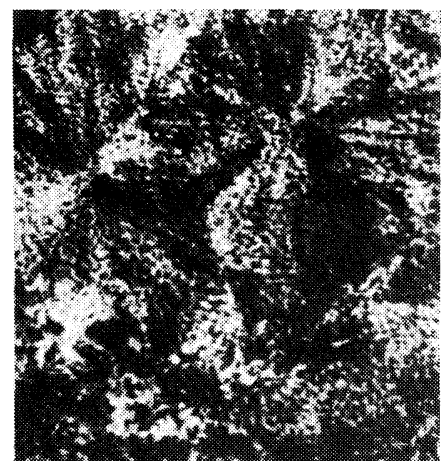

Int. contr. opt. microscopy $(\times 500) \mathrm{f}$

Figure 4. Microscopic textures of polypropylene heterophasic copolymers.

chlorine in the polymer to less than $100 \mathrm{ppm}$.

For the synthesis of EP elastomers, catalyst systems based on vanadium salts and an aluminum alkyl are generally employed. The main problem with these catalysts is their low efficiency which implies the installation of depuration sections, especially in the case of solution processes. The momentum created by the identification of high yield catalysts for PP, HDPE and linear low density polyethylene (L-LDPE), stimulated and directed our research for the identification of catalysts suited also to the production of ethylene-propylene elastomers.

The common opinion still prevails that $\mathrm{Ti}$ salt 


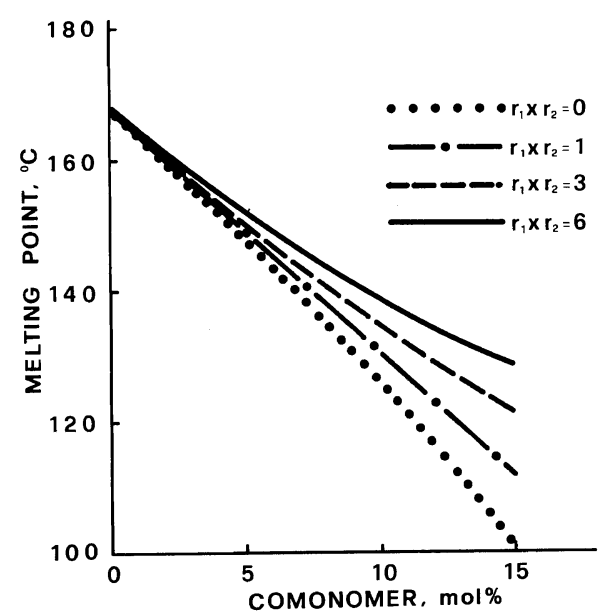

Figure 5. Effects of the ethylene content and the reactivity ratio product $r_{1} \times r_{2}$ on the melting point of polypropylene.

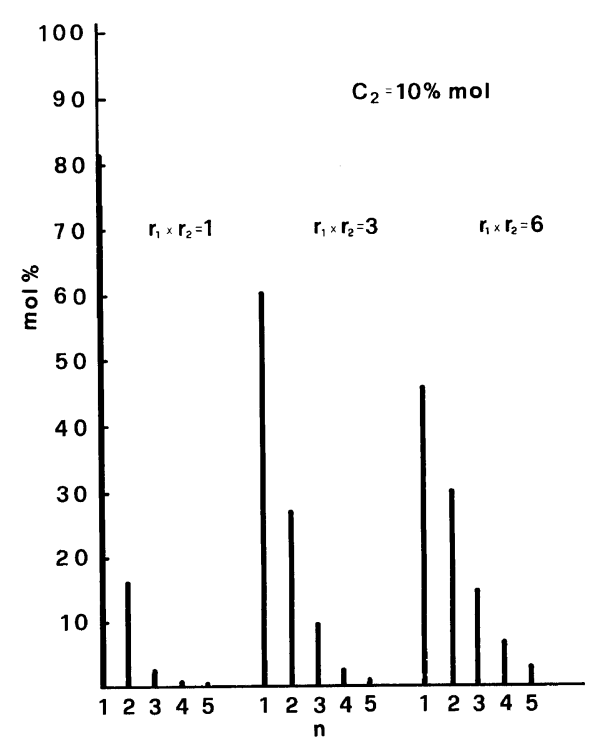

Figure 6. Theoretical distribution of the length of stereoregular sequence $n$ in statistical copolymers with various reactivity ratio product $r_{1} \times r_{2}$.

systems are not suitable for synthesizing elastomeric EP-copolymers. Nevertheless, the research efforts at ME/DUTRAL S.p.A. succeeded in identifying peculiar catalysts capable of yielding amorphous ethylene-propylene copolymers and ethylenepropylene-butene-1 terpolymers. The elastomers so produced are similar and probably better than those produced with vanadium based catalysts, at least

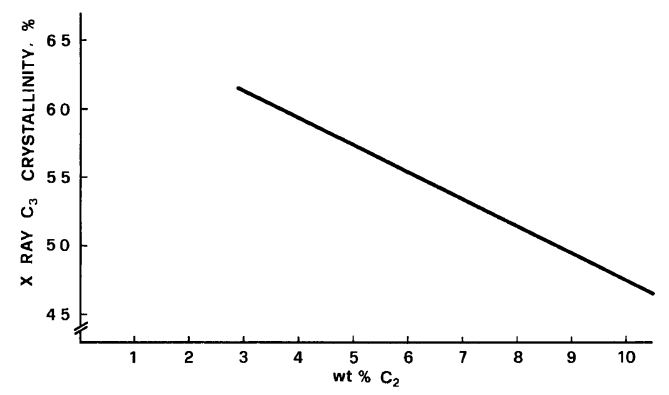

Figure 7. The lowering of crystallinity as a function of the amount of ethylene comonomer.

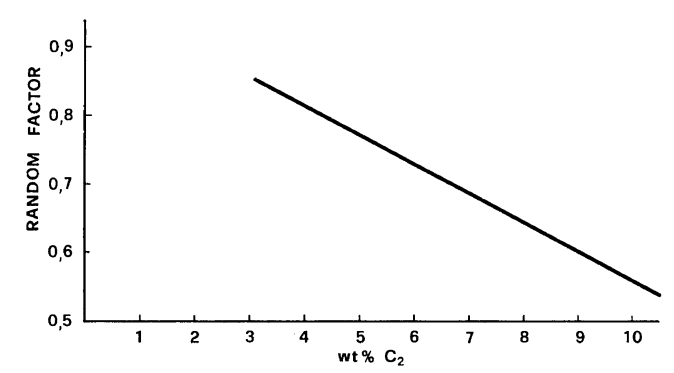

Figure 8. The random factor as a function of the amount of ethylene comonomer.

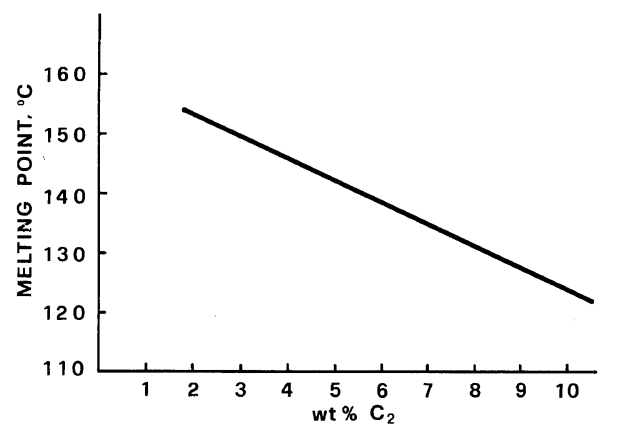

Figure 9. The melting point as a function of the amount of ethylene comonomer.

for some applications. Furthermore these Ti catalysts are able to work in a heterogeneous reaction medium, i.e., in a suspension process in use at DUTRAL S.p. A. On the contrary, as it is known, most EPM/EPDM elastomer manufacturers are currently employing solution processes. The reaction medium in such processes is a solvent to which ethylene, propylene and the catalyst are added. The end-product is generally recovered from the solution. The flow sheet of this process is shown 


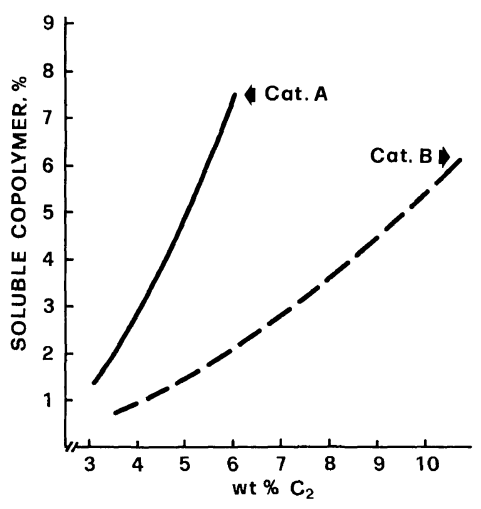

Figure 10. The amount of amorphous fraction in two series of polypropylene statistical copolymers with ethylene.

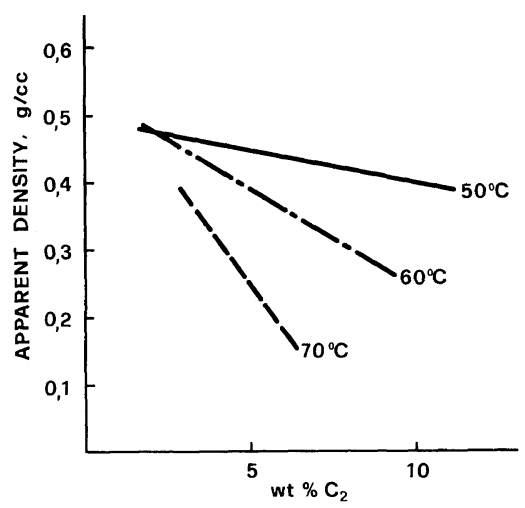

Figure 11. The apparent density of polypropylene copolymers after extraction of soluble fractions at various temperatures.

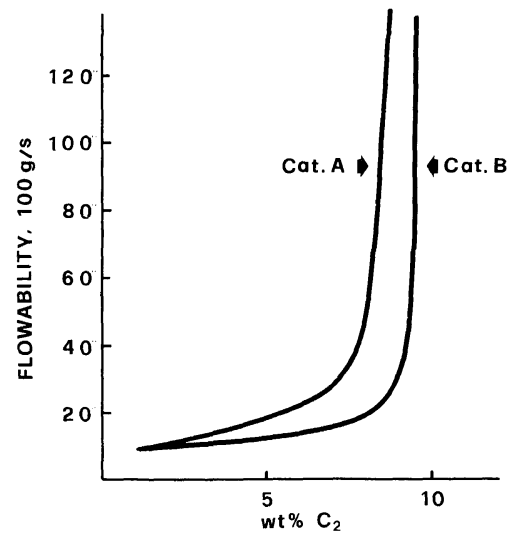

Figure 12. The flowability of powder polymers.

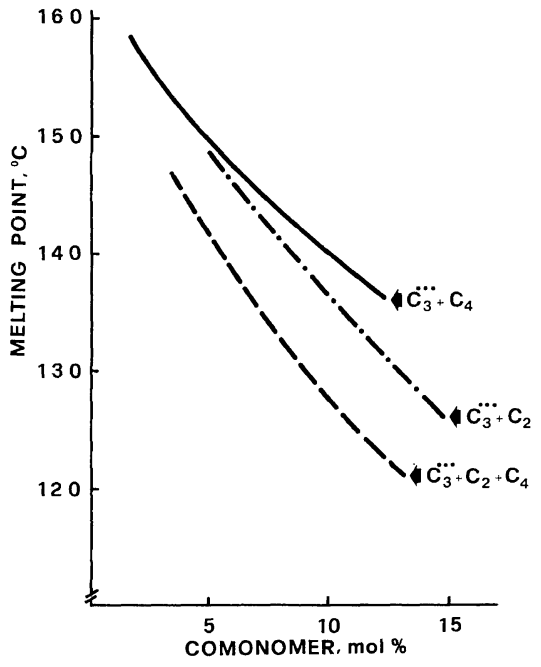

Figure 13. The melting points of polypropylenes modified by bipolymerization with ethylene or butene and by terpolymerization with ethylene and butene.

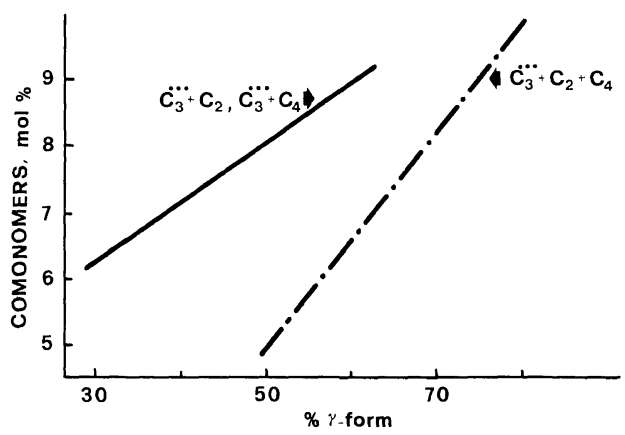

Figure 14. Variation of the crystalline form of polypropylene due to bipolymerization or terpolymerization with ethylene and butene.

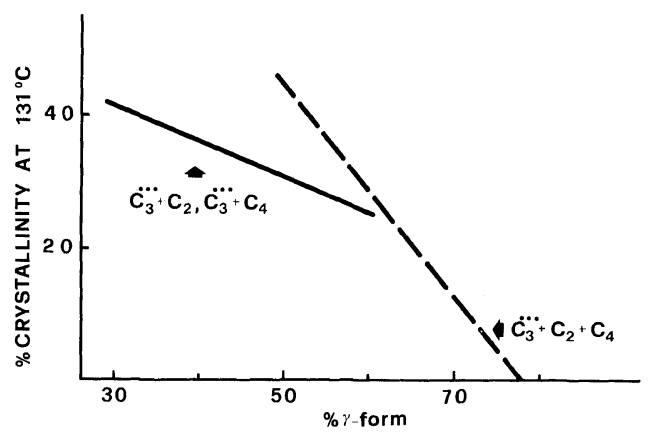

Figure 15. Relation between the crystallinity and the crystalline form. 


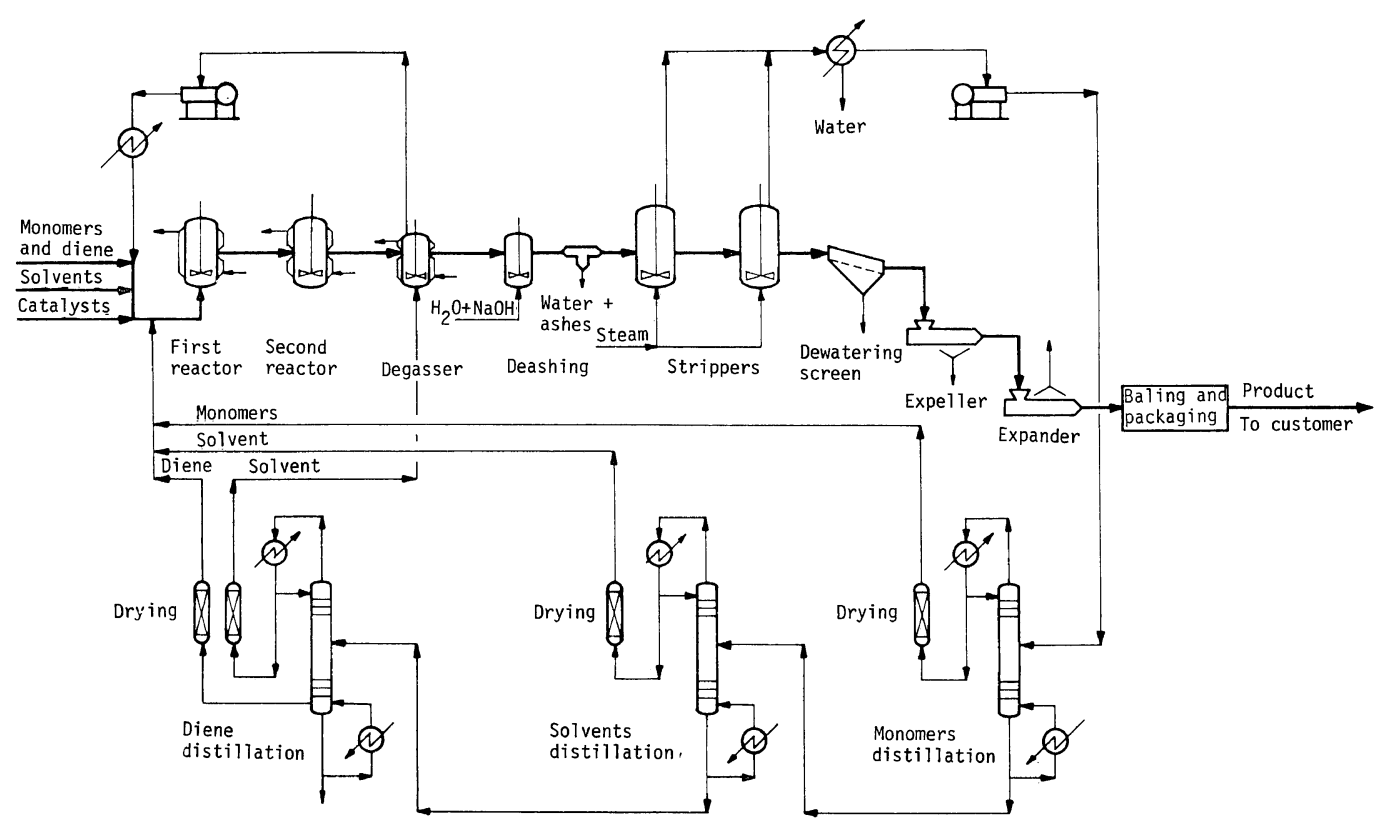

Figure 16. The solution process for ethylene-propylene-rubber (EPR) production.

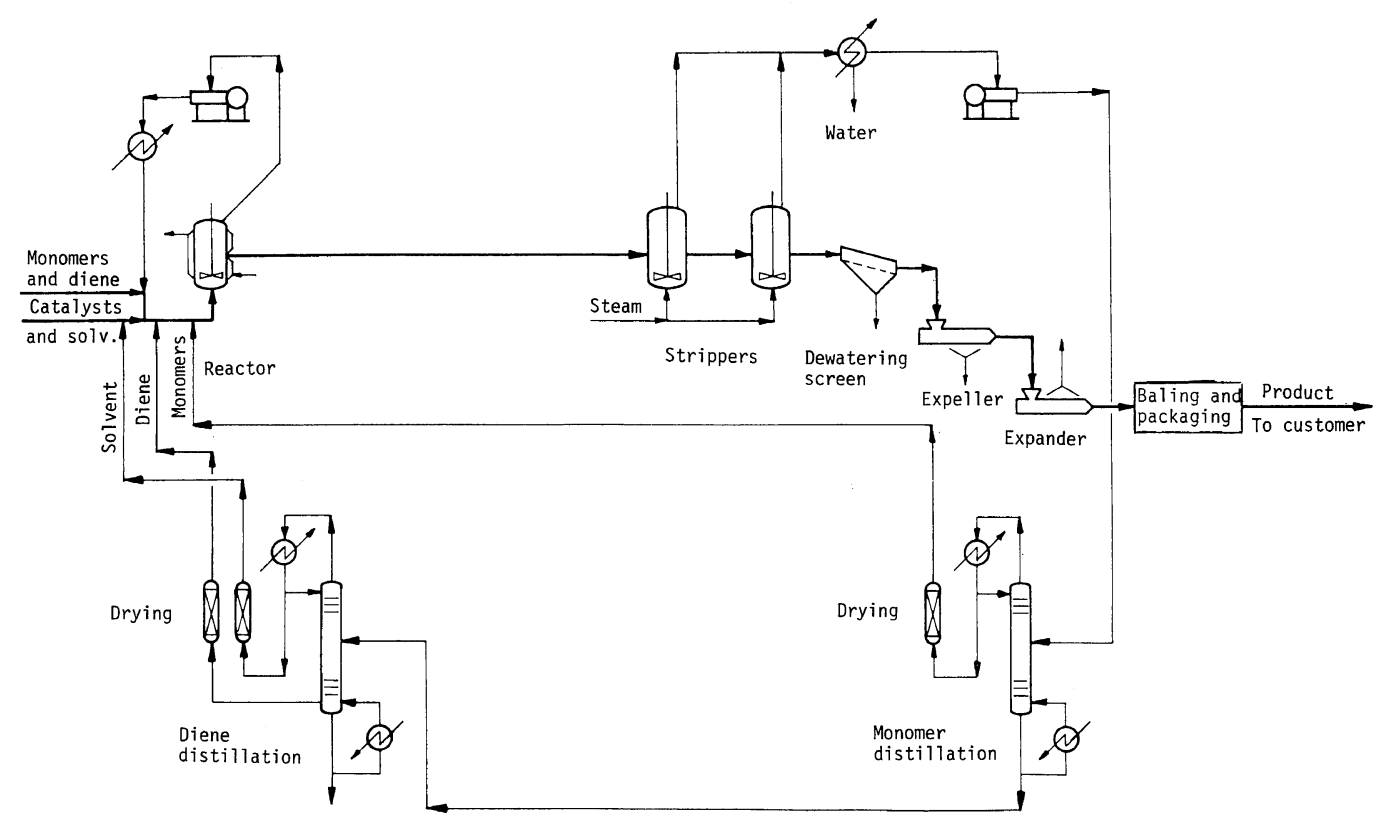

Figure 17. The DUTRAL suspension process for EPR production.

in Figure 16. However, ME/DUTRAL S.p. A. has are added. The polymer is produced as fine particles preferred from the beginning a liquid propylene suspended in the liquid mixture. The flow sheet in reaction medium to which ethylene and the catalyst Figure 17 shows how this process is much simpler 
than the solution processes. In addition, there are a number of advantages: (a) No stirring viscosity problems, since the polymer is not dissolved into the polymerization medium; (b) easy removal of the reaction heat by vaporizing the monomers partially; they are then recycled by a compressioncondensation process; (c) a very homogeneous reaction medium because its low viscosity allows an intense evaporation and leads to a fast turnover in the reactor and an excellent control of the molar ratio of the monomers in the polymer; (d) high concentration of the polymer in the medium (up to $35 \%$ by weight) which means a high process productivity; (e) high polymerization yields (20 to $50 \times 10^{3} \mathrm{~g}$ polymer/g V) which allows the purification from catalyst residues to be partially simplified with respect to the solution processes; (f) the capability of producing very high MW polymers; (g) lower investment and production costs.

Figures 18 and 19 illustrate the lower costs of the suspension process. It is worthwhile to point out that the quality of the product is not all sacrificed. In fact, the composition of the polymers is more constant and homogeneous with respect to those obtained with the solution process. This constant quality translates into the constant rheological characteristics of polymers and their compounds during the various phases of the transformation process and the constant mechanical properties of cured compounds.

The superiority of the suspension process will now be boosted by the truly happy marriage with $\mathrm{Ti}$ catalysts. In fact some suitable high yield catalysts fit perfectly the suspension process without wasting their fine performances, including replica phenomena.

As can be seen from Figure 20, the degree of crystallinity changes according to the type of supported catalyst used. In our case, we have indicated three different types of catalyst as A, B and C, although we cannot describe in detail for evident reasons.

As demonstrated by ${ }^{13} \mathrm{C}$ NMR analysis, ${ }^{15}$ copolymers obtained from titanium catalysts are free from inversions and have $r_{1} \times r_{2}>1$ for type $\mathrm{A}$ and

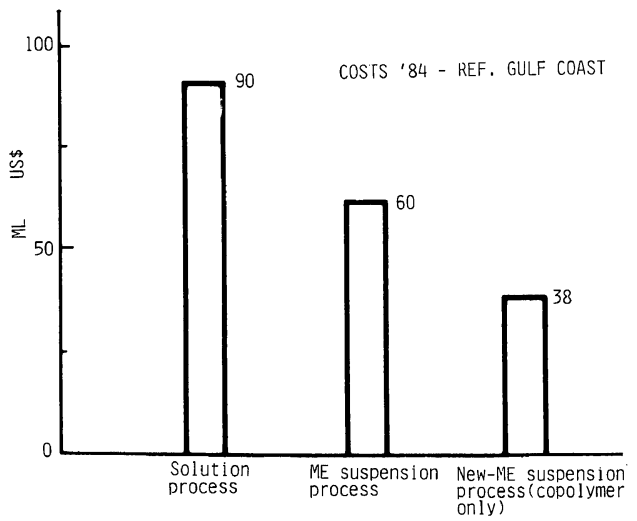

Figure 18. Total investment costs of EPR production plant (nominal capacity 45,000 tons/year. grass roots basis).

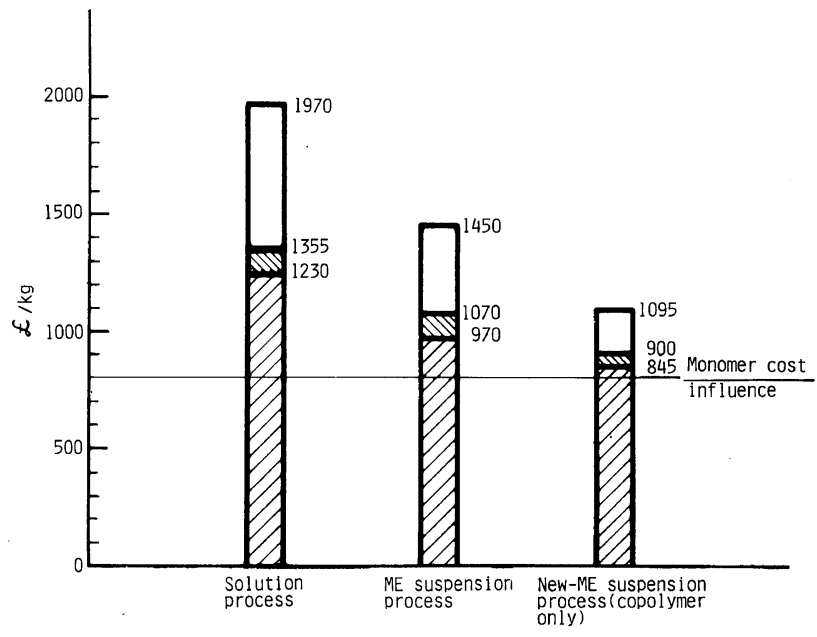

Figure 19. EPR total production costs (45,000 tons/year). $\square$, indirect operating costs and return on fixed capital investment; $\mathbb{Q}$ labor-maintenance, ---; $\square$, raw materials and utilities; (cost 1984). 


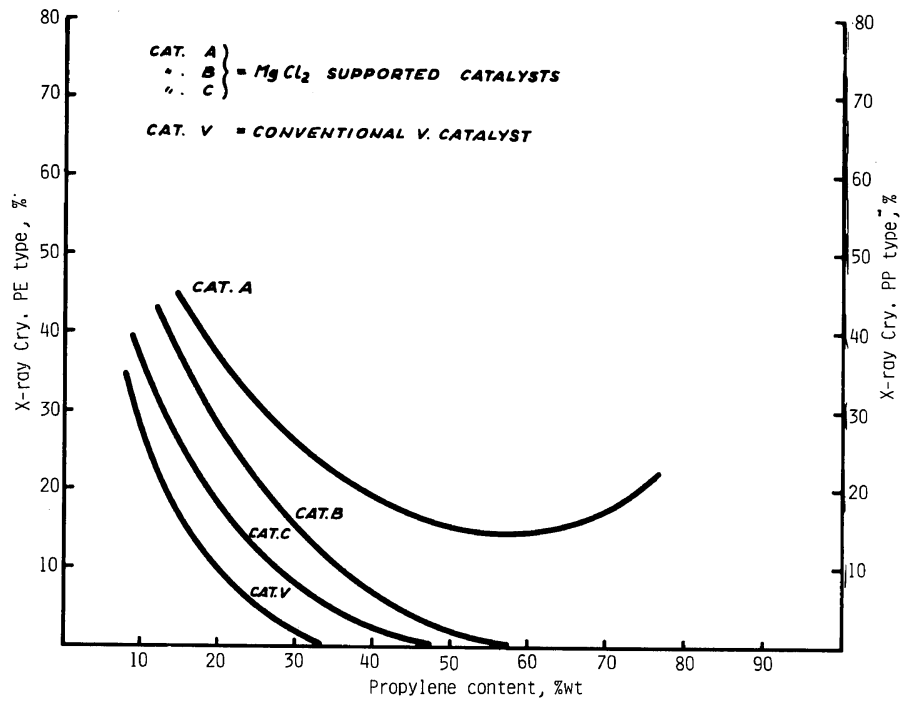

Figure 20. Polyethylene or polypropylene crystallinity content (from X-rays analysis).

B catalysts and $r_{1} \times r_{2} \cong 1$ for the type C catalysts, whereas the product from vanadium-based catalyst contains a considerable amount of inversions (about $15 \%$ ) and has $r_{1} \times r_{2}<1$. This proves that from catalyst $\mathrm{A}, \mathrm{B}, \mathrm{C}$ to $\mathrm{V}$ there is an increasing reduction of blocking of propylenic and ethylenic type sequences. Figure 21 reports molecular weight distribution (MWD) of products obtained with titanium salt supported catalysts in comparison with a similar product obtained with vanadium catalyst. All high yield products have broader MWD and the broadest is that of the products of the catalyst $\mathbf{B}$.

Figure 22 shows the behaviour of the melt viscosity as a function of the shear rate. It can be seen that high yield products have a greater pseudoplasticity probably due to their broader MWD. Figure 23 shows the behaviour of the zero-shear Newtonian viscosity, determined with a Weissenberg rheogoniometer, as a function of the average viscosimetric molecular weight $\left(M_{v}\right)$. $\log \eta_{0} / \log M_{v}$ correlations for the three families of products are practically the same.

\section{Polymer Features}

Composition and Mooney viscosity of the polymers produced are located in the hatched area in Figure 24. These polymers have been compared to copolymers obtained with vanadium catalyst and

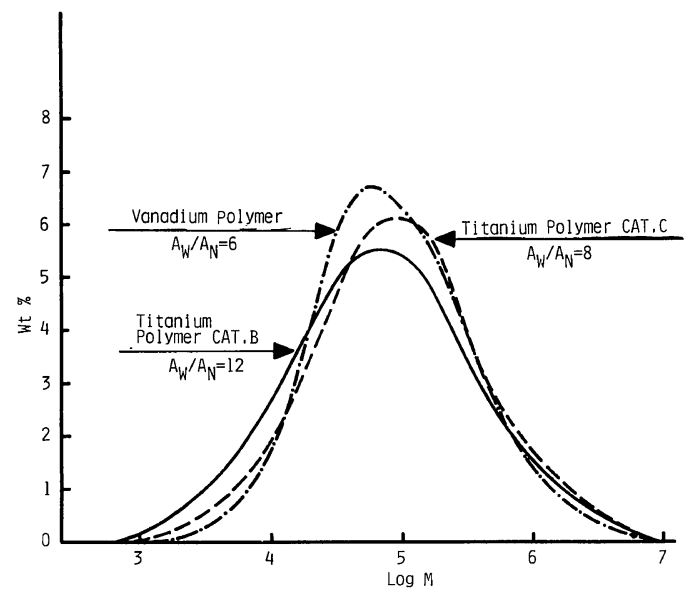

Figure 21. Comparison of molecular weight distribution of some EPR copolymers. --.--, CAT. V conventional vanadium catalysts; ---, CAT. C $\mathrm{MgCl}_{2}$ supported catalysts; - - CAT. B $\mathrm{MgCl}_{2}$ supported catalysts.

currently sold on the market such as DUTRAL CO054, CO-059, CO-034,, CO-038. The reference parameters were propylene content, Mooney viscosity and green strength which reflects the market demands better. The strength of polymers (with identical elastic properties, same propylene content and Mooney viscosity) measured at $100 \%$ elongation and at breaking point does not differ significantly 
New Trends in Propylene Based Polymer Production

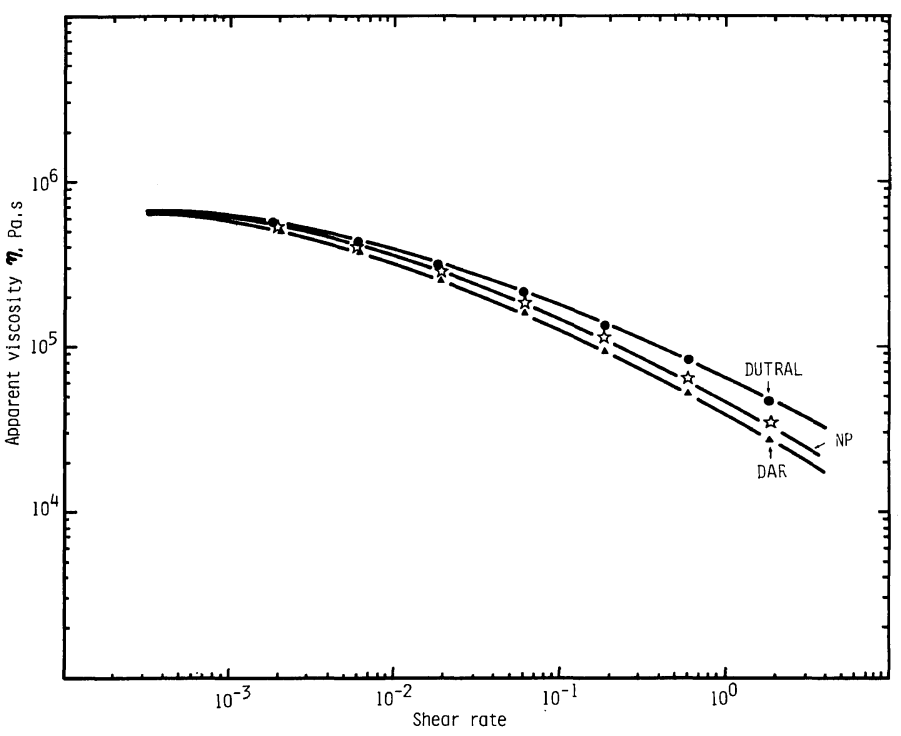

Figure 22. Apparent viscosty as fuctions of the shear rate at $150^{\circ} \mathrm{C}$. DUTRAL, copolymer obtained with conventional vanadium catalyst; $\mathrm{NP}$, copolymer obtained with cat.c.; $\triangle \mathrm{DAR}$, copolymer obtained with cat. b.

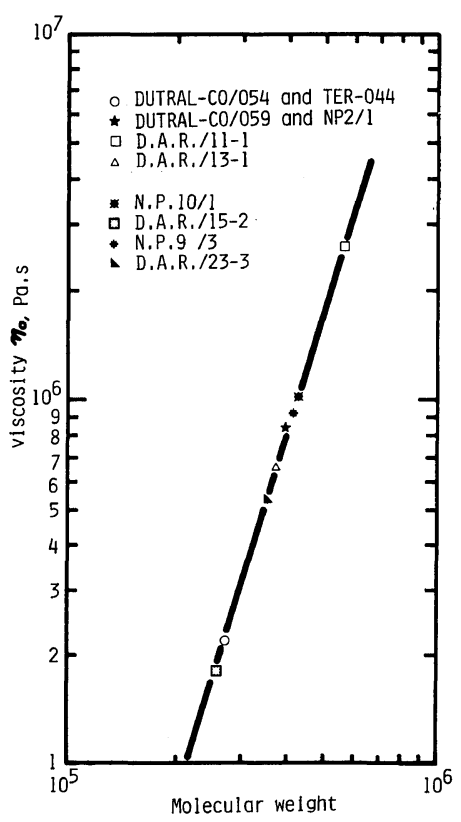

Figure 23. Zero-shear (Newtonian) viscosity at $150^{\circ} \mathrm{C}$ as a function of molecular weight. $\log \eta_{0}=-12.9+3.36$ $\log M_{v}$. DUTRAL, copolymer obtained with conventional vanadium catalyst; NP, copolymer obtained with CAT. C; DAR, copolymer obtained with CAT. B. from that of the traditional polymers. An interesting comparison of the stress-strain curves is shown in Figures 25 and 26. Their shape is strictly determined by the structure of the polymers and MWD: with a wide MWD the polymer is amorphous and the curves approach to those of cured mixtures in their shape. Comparable resistance can be reached in the case of polymers with a higher green strength containing variable quantities of propylene. Their higher resistance depends on the larger sequences of ethylene which start forming crystalline aggregates. This different behaviour is in agreement with the results obtained with ${ }^{13} \mathrm{C}$ NMR studies. This means that, for the above mentioned C-type catalyst, propylene content of approximately $35-37 \%$ by weight is necessarry to yield a measurable amount of crystallized polyethylene chains. With vanadium-catalysts, the same type of copolymers are obtained with propylene content of 28 $30 \%$ by wt. Therefore, the price difference between ethylene and propylene (propylene is cheaper) makes Ti catalysts more economical. Stress-strain curves are compared in Figure 27. The wider MWD in high yield polymers renders the shape of the curves more similar to cured mixtures. 


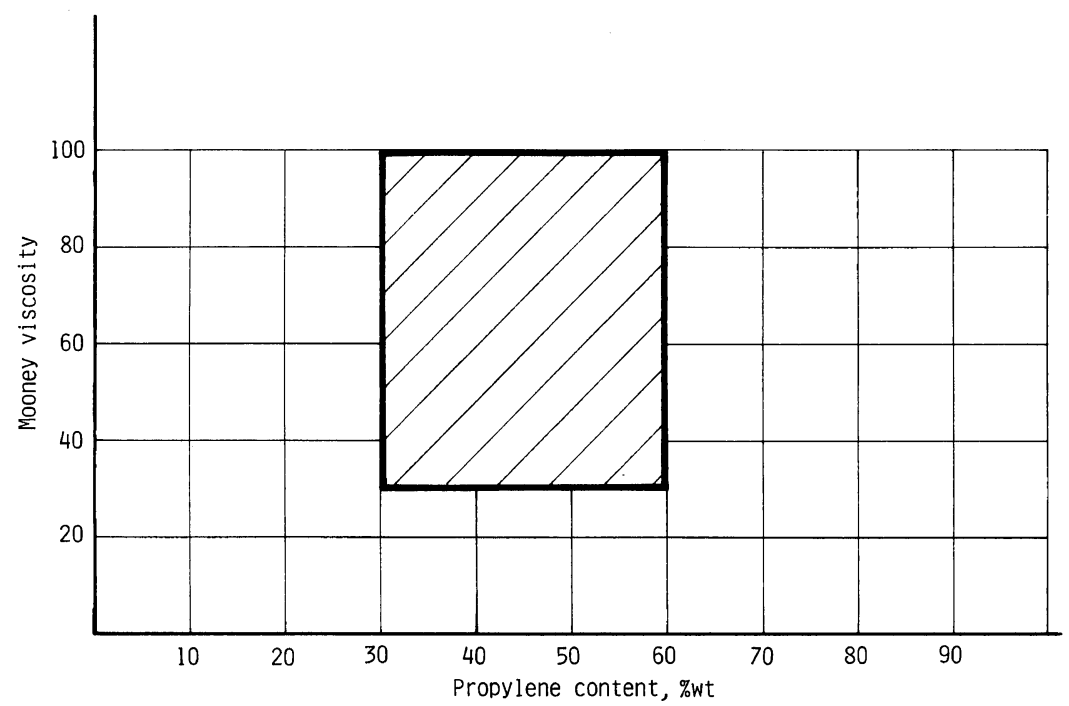

Figure 24. Explored area in titanium catalyst polymerization of ethylene-propylene copolymers, as expressed by the Mooney viscosity $\mathrm{ML}(1+4)$ at $100^{\circ} \mathrm{C}$ and the propylene content.

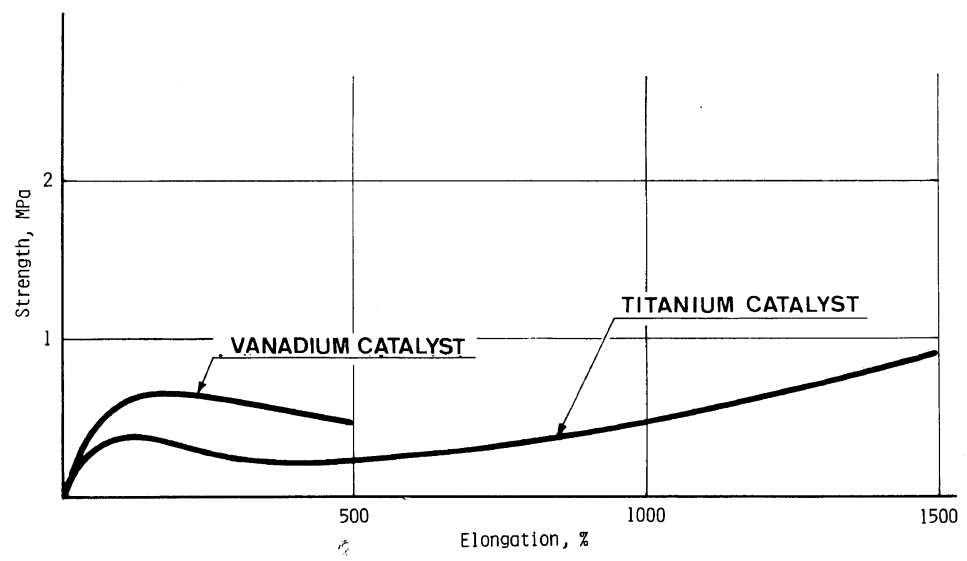

Figure 25. Stress-strain properties of a raw ethylene-propylene elastomer (DUTRAL CO 054-low green strength grade).

\section{Elastomeric Properties at Low Temperatures}

Differential scanning calorimetry (DSC) analysis surprisingly showed that the glass transition temperature of this new class of copolymers is a couple of degrees lower than that of $\mathrm{V}$ ones (Table I, Figure 28). The superior behaviour of Ti copolymers was also confirmed by TR-50 measurements on cured items (Table II). Furthermore, by terpolymerizing ethylene-propylene-butene- 1 and by determining the glass transition temperature with dilatometry a significant decrease (Table III) of this value was shown substantiating the hypothesis of Davison, Taylor and Seidov ${ }^{16,17}$ concerning the possibility of increasing elastomeric properties; i.e., decreasing the glass transition temperature, by adding a termonomer.

\section{Mechanical Properties}

Cured items of $\mathrm{Ti}$ and $\mathrm{V}$ copolymers have very similar mechanical properties. The tensile strength increases with the propylene content (Figure 29). By adding butene- 1 to the copolymer until the $\mathrm{C} 3 / \mathrm{C} 4$ 
New Trends in Propylene Based Polymer Production

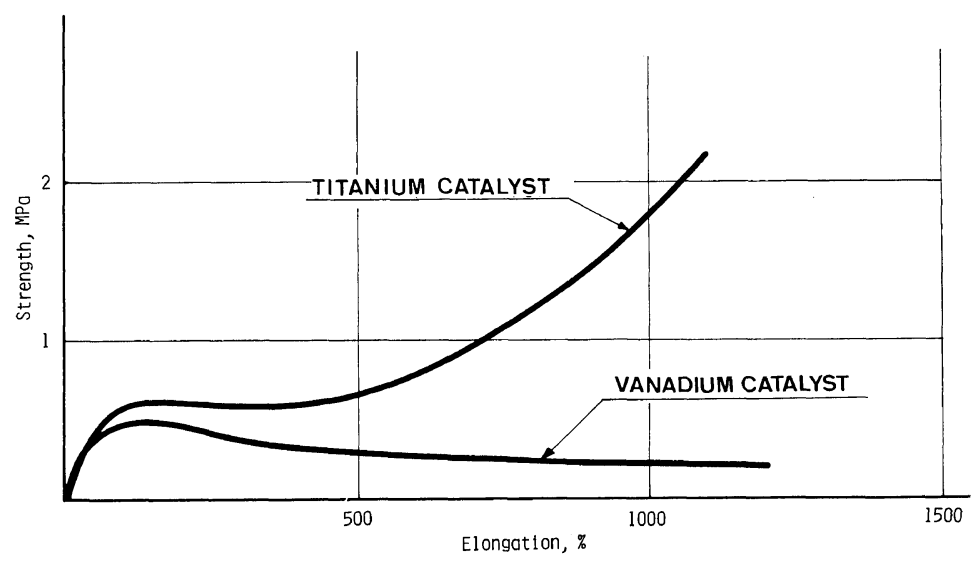

Figure 26. Stress-strain properties of a raw ethylene-propylene elastomer (DUTRAL CO 059-low green strength grade).

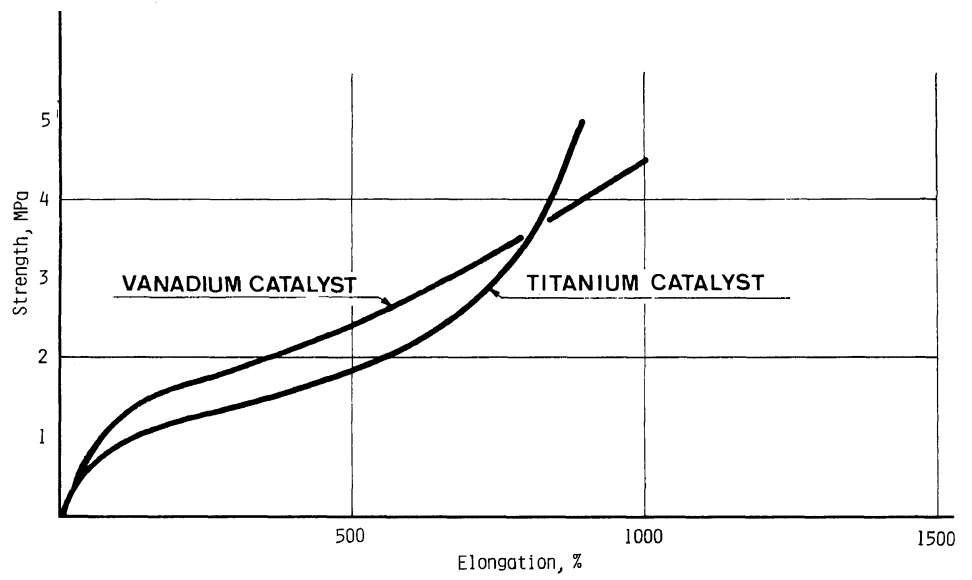

Figure 27. Stress-strain properties of a raw ethylene-propylene elastomer (DUTRAL CO 038-high green strength grade).

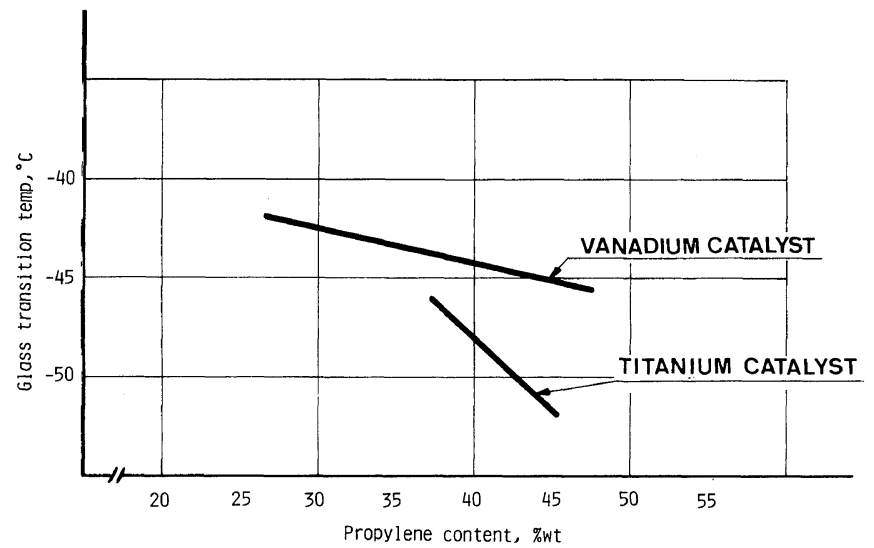

Figure 28. Glass transition temperature (DSC method) versus propylene content for ethylene-propylene elastomers. 
Table I. Ethylene-propylene elastomers glass transition temperature DSC method

\begin{tabular}{|c|c|c|}
\hline \multirow{3}{*}{ Grade } & \multicolumn{2}{|c|}{ Catalyst } \\
\hline & Titanium & Vanadium \\
\hline & ${ }^{\circ} \mathrm{C}$ & ${ }^{\circ} \mathrm{C}$ \\
\hline DUTRAL CO 054 & -50 & -44 \\
\hline DUTRAL CO 059 & -50 & -45 \\
\hline DUTRAL CO 034 & -48 & -42 \\
\hline DUTRAL CO 038 & -47 & -43 \\
\hline
\end{tabular}

Table II. Ethylene-propylene elastomers TR 50 on crosslinked compounds (TR Test-ASTM D 1329)

\begin{tabular}{|c|c|c|}
\hline \multirow{3}{*}{ Grade } & \multicolumn{2}{|c|}{ Catalyst } \\
\hline & Titanium & Vanadium \\
\hline & ${ }^{\circ} \mathrm{C}$ & ${ }^{\circ} \mathrm{C}$ \\
\hline DUTRAL CO 054 & -18 & -14 \\
\hline DUTRAL CO 059 & -18 & -15 \\
\hline DUTRAL CO 034 & -15 & -5 \\
\hline DUTRAL CO 038 & -15 & -10 \\
\hline
\end{tabular}

Table III. Chemical-physical characteristics and $T_{\mathrm{g}}$ dilatometric values

\begin{tabular}{|c|c|c|c|c|c|}
\hline \multirow{2}{*}{ Type of products } & {$[\eta]$} & $\mathrm{C}_{3}$ & $\mathrm{C}_{4}$ & $\%$ crystalline & $T_{\mathrm{g}}$ \\
\hline & $\mathrm{dlg}^{-1}$ & $w t \%$ & $\mathrm{wt} \%$ & PE type & ${ }^{\circ} \mathrm{C}$ \\
\hline $\begin{array}{l}\text { DUTRAL CO } 059 \\
\text { V. catalyst }\end{array}$ & 3.00 & 43 & - & 0 & -55 \\
\hline $\begin{array}{l}\text { EP copolymer } \\
\text { Ti catalyst, type B }\end{array}$ & 2.80 & 50 & - & 3 & -51 \\
\hline $\begin{array}{l}\text { EP copolymer } \\
\text { Ti catalyst, type C }\end{array}$ & 3.10 & 44 & - & 0 & -54 \\
\hline $\begin{array}{l}\text { EP copolymer } \\
\text { Ti catalyst, type C }\end{array}$ & 3.40 & 39 & - & 2 & -51 \\
\hline $\begin{array}{l}\text { Ethylene-propylene- } \\
\text { butene-1 }\end{array}$ & 2.80 & 20 & 28 & 0 & -61 \\
\hline Ti catalyst, type $\mathrm{C}$ & 2.40 & 31 & 38 & 0 & -61 \\
\hline Ti catalyst, type C & 3.10 & 23 & 17 & 1 & -57 \\
\hline Ti catalyst, type $\mathrm{C}$ & 4.10 & 20 & 12 & 4 & -56 \\
\hline
\end{tabular}

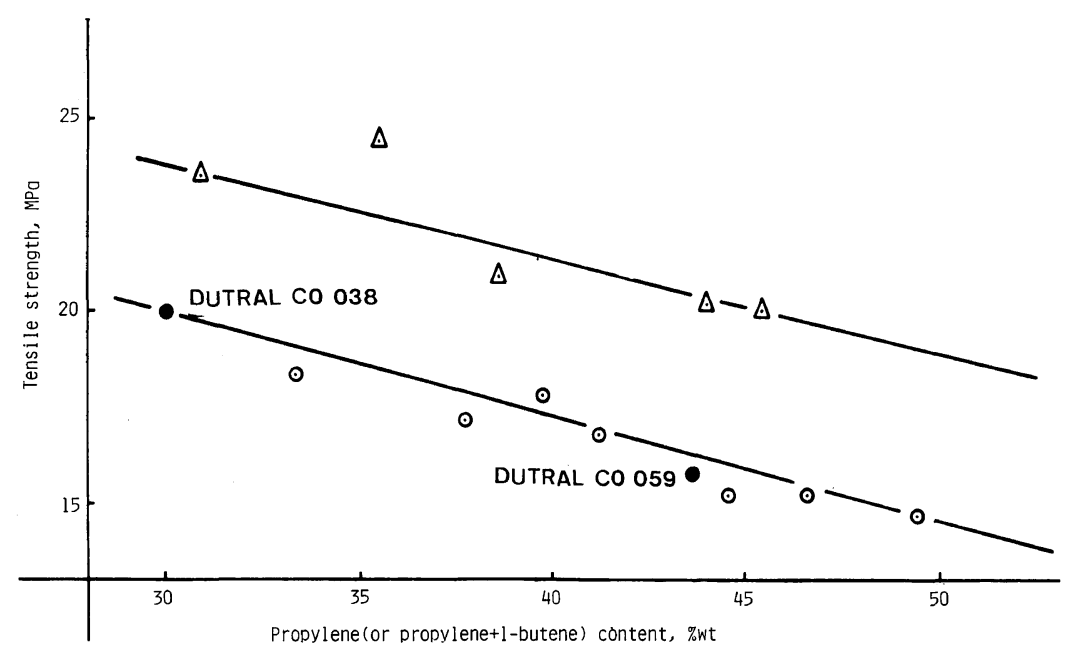

Figure 29. Tensile strength of cured EPR copolymers. Circles represent $C_{2}-C_{3}$ copolymers obtained with catalyst $C$, and triangles represent $\mathrm{C}_{2}-\mathrm{C}_{3}-\mathrm{C}_{4}$ terpolymers obtained with catalyst $\mathrm{C}$. 


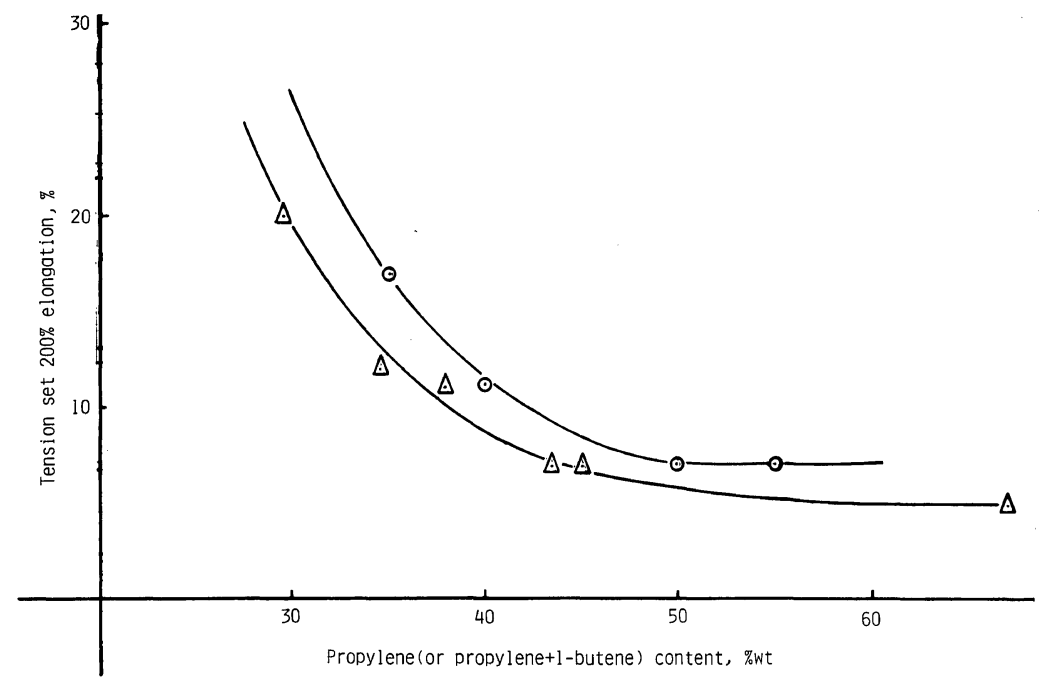

Figure 30. Tension set $200 \%$-elongation at room temperature of cured EPR copolymers. The circles and triangles are the same as in Figure 29.

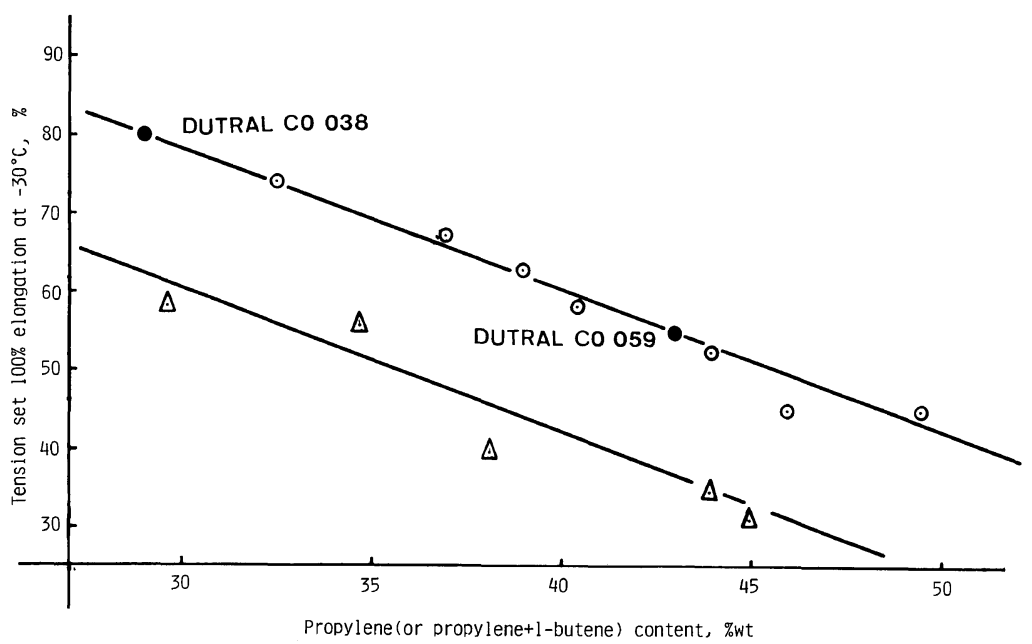

Figure 31. Tension set $100 \%$ elongation for $30 \mathrm{~min}$ at $-30^{\circ} \mathrm{C}$, reading at $-30^{\circ} \mathrm{C} 10 \mathrm{~min}$ after release. The marks are the same as in Figure 29.

molar ratio is approximately 1 , a significant increase in the tensile strength is obtained for cured items (Figure 29) accompanied by a decrease in tension set at $200 \%$ elongation to values comparable to the best copolymers obtained with $\mathrm{V}$ salt based catalysts (Figure 30). Furthermore, a significant decrease in tension set at $-30^{\circ} \mathrm{C}$ at $100 \%$ elongation indicates a superior behaviour of cured items at low temperatures (Figure 31). In fact, under these conditions, they behave better than V-EP polymers as well. Cured items are generally superior to their $\mathrm{V}$ counterparts at high temperatures as well, a property to be expected for their high chemical purity. In fact, high yield co- and ter-polymers show a residual chlorine lower than $50 \mathrm{ppm}$ and a total ash content lower than $200 \mathrm{ppm}$. Their higher resistance at high temperatures is illustrated in Figures 32 and 33 , which show the tensile strength and elongation at break at $150^{\circ} \mathrm{C}$. The recipe and characteristics of the cured items are indicated in Table IV. 


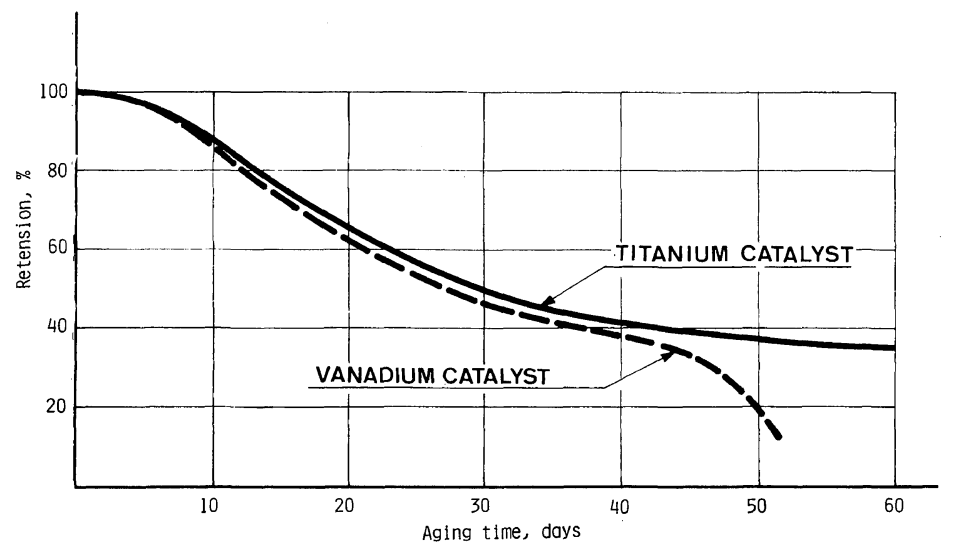

Figure 32. Retention of the tensile strength for a cured EPR copolymer (DUTRAL CO 038) as a function after oven aging at $150^{\circ} \mathrm{C}$ (Formulation in Table IV).

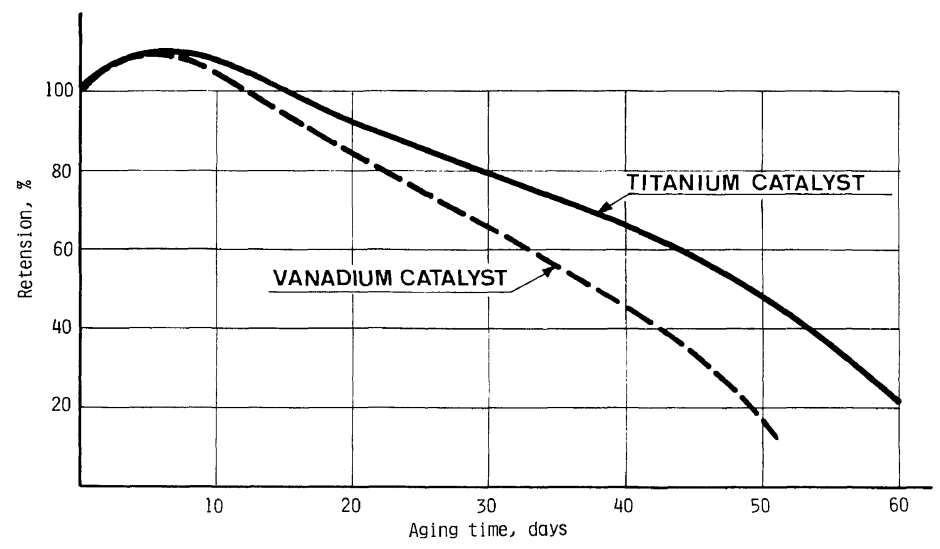

Figure 33. Retention of the elongation at break for a cured EPR (DUTRAL CO 038) after oven aging at $150^{\circ} \mathrm{C}$ (Formulation in Table IV).

Table IV. Ethylene-propylene elastomers, DUTRAL CO 038 formulation for aging test (Figures 32 and 33)

\begin{tabular}{|c|c|c|}
\hline Polymer & \multicolumn{2}{|c|}{100} \\
\hline Zinc oxide & \multicolumn{2}{|c|}{5} \\
\hline Stearic acid & \multicolumn{2}{|c|}{1} \\
\hline FEF Black & \multicolumn{2}{|c|}{80} \\
\hline Paraffinic oil & \multicolumn{2}{|c|}{30} \\
\hline MB & \multicolumn{2}{|c|}{2} \\
\hline Anox & \multicolumn{2}{|c|}{0.5} \\
\hline Sulphur & \multicolumn{2}{|c|}{0.3} \\
\hline Peroximon F. 40 & \multicolumn{2}{|c|}{4.5} \\
\hline Curing at $165^{\circ} \mathrm{C}$ for $40^{\prime}$ & & \\
\hline $\begin{array}{l}\text { Mechanical properties } \\
\text { of cured compound }\end{array}$ & $\begin{array}{c}\text { Titanium } \\
\text { catalyst }\end{array}$ & $\begin{array}{l}\text { Vanadium } \\
\text { catalyst }\end{array}$ \\
\hline Tensile strength/MPa & 16.2 & 16.3 \\
\hline Elongation at break $/ \%$ & 420 & 400 \\
\hline
\end{tabular}

Table V. Ethylene-propylene Elastomers dielectric characteristics

\begin{tabular}{lcc}
\hline & $\begin{array}{c}\text { Titanium } \\
\text { catalyst }\end{array}$ & $\begin{array}{c}\text { Vanadium } \\
\text { catalyst }\end{array}$ \\
\hline $\begin{array}{c}\text { Dielectric constant } \\
(1000 \mathrm{~Hz})\end{array}$ & $1.8-1.9$ & $1.9-2.0$ \\
$\begin{array}{c}\text { Dielectric breakdown/ } \\
\mathrm{kV} \mathrm{mm} \mathrm{mm}^{-1}\end{array}$ & $25-29$ & $24-28$ \\
$\begin{array}{c}\text { Volume resistivity/ } \\
\Omega \mathrm{cm}^{-1}\end{array}$ & $1.5-1.7 \times 10^{7}$ & $1.3-1.6 \times 10^{7}$ \\
\hline
\end{tabular}

\section{Dielectric Properties}

The dielectric properties of co- and ter-polymers obtained with high yield catalysts are shown in Table V. Unsurprisingly, their greater purity renders them better dielectrics than $\mathrm{V}$ copolymers. 


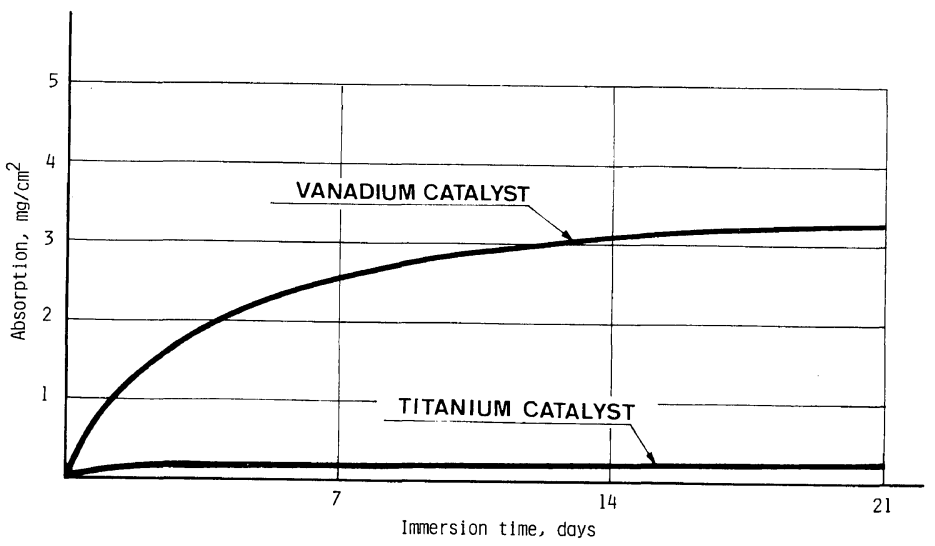

Figure 34. Water absorption of pure crosslinked EPR copolymer (DUTRAL CO 054) after immersion in water at $100^{\circ} \mathrm{C}$.

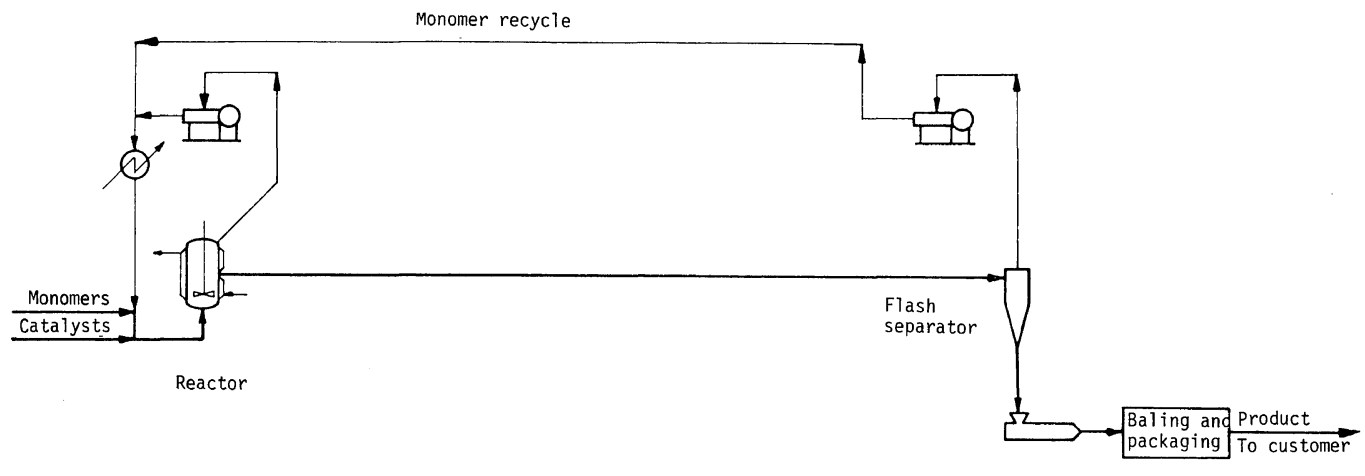

Pelletizing

Figure 35. New DUTRAL suspension process for EPR production.

The new products are also more stable in water, a very important characteristic when dealing with electrical cables installed in a damp environment. The laboratory test consists of keeping a cured polymer devoid of electrical charges in water at $100^{\circ} \mathrm{C}$. The quantity of water absorbed by the sample is measured at regular intervals and referred to the total surface exposed to water. Figure 34 shows the water absorbtion curves of two types of DUTRAL CO-054 exposed to water for 21 days. The resistance to currrent leaks for ethylenepropylene elastomers is very high. The same is true for treeing, an electrochemical alteration due mainly to water which in time leads to perforation of the insulation. Treeing is relatively frequent when polyethylene insulation is used for medium or high tension cables.

\section{Applications}

Polymers synthesized on high yield catalysts represent the better alternative and can substitute the traditional ones in all the application fields which employ unsaturated terpolymers for technical or economical reasons. In technical applications Ti coand ter-polymers have the advantage of a superior behaviour at low temperatures and a higher resistance at high ones. The very low content in catalyst residues makes them ideal for atoxic items. Our tests confirmed their excellent stability in damp environments, even in very severe conditions, which makes them the best suited insultators for electrical cables. Although co- and ter-polymers, ethylenepropylene and ethylene-propylene-butene-1 already are excellent insulators, the quality of insulating recipe will be further improved making them 
even more reliable and suitable for voltages higher than those currently employed.

Since copolymers obtained with high yield catalysts are at least as good as those produced with vanadium catalysts one can move directly to the manufacture of EP elastomers with high yield catalysts employing the ME/DUTRAL S.p.A. suspension process. At last, dramatic simplification of the process is made possible by the very high yield and by the replica phenomenon. In fact, polymers can be obtained with no need of the usual stripping and drying operations indispensable to the current vanadium catalyst ME/DUTRAL S.p.A. process. One way of representing the new simplified process is illustrated in Figure 35.

The following steps are contemplated: (1) Suspension polymerization in liquid monomers or monomer mixtures with aliphatic hydrocarbons with a boiling point lower than $+10^{\circ} \mathrm{C}$ (propane, butane, etc.); (2) flash of the reaction medium with evaporation of monomers and diluents; (3) formation of copolymer elastomers in powder, flakes or bales.

This simplified process involves lower investment and lower production costs. In fact, in a 45,000 tons/year plant it would allow a cut of about $50 \%$ on the investment expenses necessary with the traditional suspension process (Figure 18). The need to periodically clean-up the reactor, a drawback of processes employing traditional catalysts, is significantly reduced with the new process thanks to the different structure of the high yield elastomers. The polymer obtained with the traditional process is made of powder specks of random shapes (Figures 36 and 37). The particles of the polymer from high

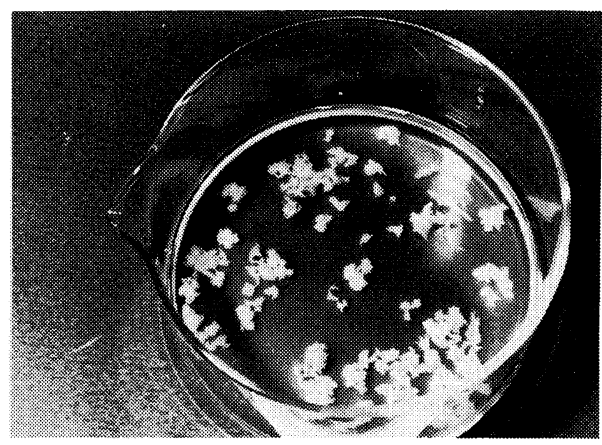

Figure 36. Copolymer obtained with the conventional vanadium catalyst (methanol-acetone mixture, $1: 1 \mathrm{v} / \mathrm{v}$ ). yield catalysts have a well defined regular shape and their sizes, much more evenly distributed, range from 0.5 to $1 \mathrm{~mm}$ (Figures 38, 39, and 40). This makes possible the use of higher concentrations of rubbery suspension and improves its processability.

Our research proves that when "particle form"

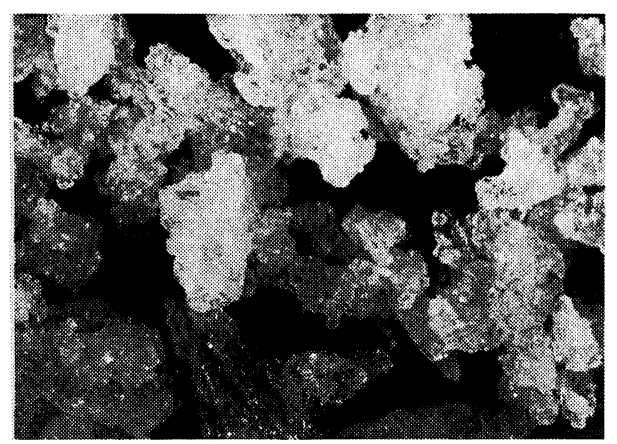

Figure 37. A particular particle form copolymer $(\times 10)$ obtained with the conventional vanadium catalyst.

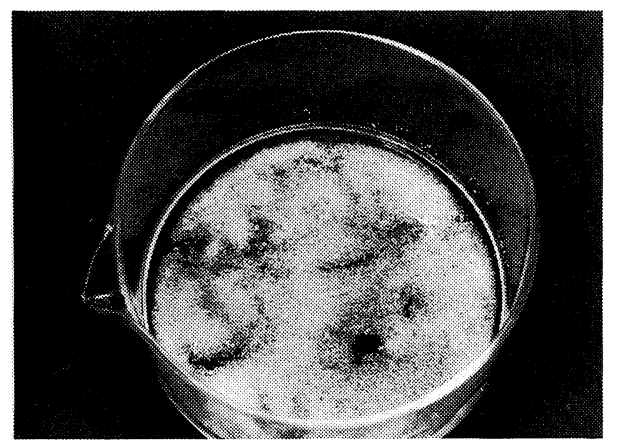

Figure 38. Copolymer obtained with the $\mathrm{MgCl}_{2}$ supported catalyst type $\mathrm{C}$ (methanol-acetone mixture, $1: 1$ $\mathrm{v} / \mathrm{v})$.

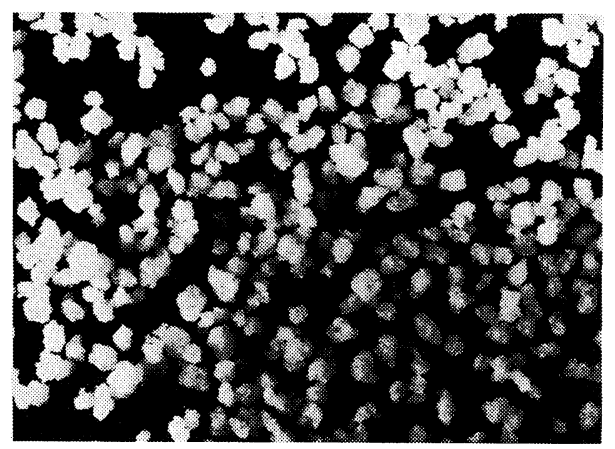

Figure 39. A particular particle form copolymer $(\times 10)$ obtained with the $\mathrm{MgCl}_{2}$ supported catalyst type $\mathrm{C}$. 


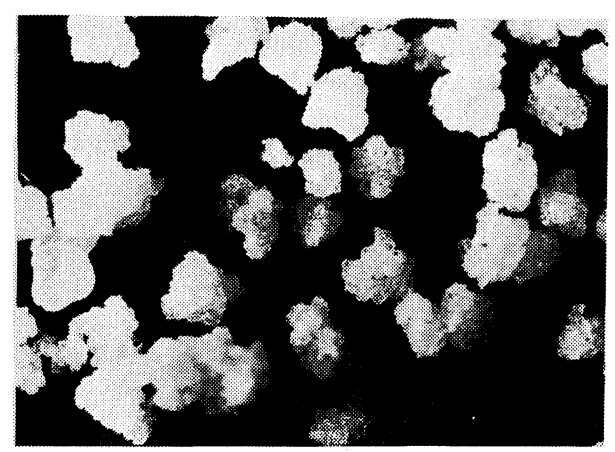

Figure 40. A particular particle form copolymer $(\times 25)$ obtained with the $\mathrm{MgCl}_{2}$ supported catalyst type $\mathrm{C}$.

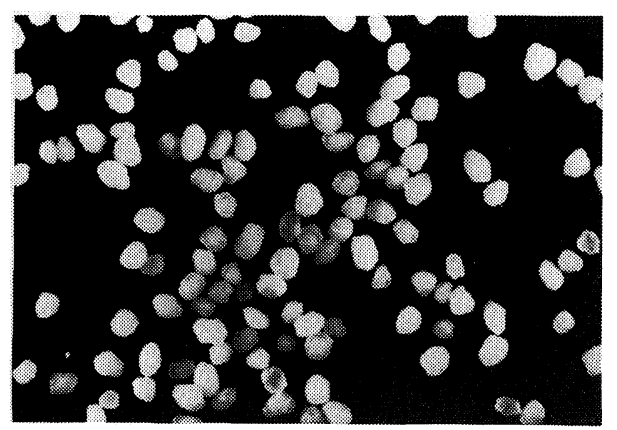

Figure 41. A particular particle form catalyst $(\times 25)$ (catalyst type $\mathrm{C}$ is supported on organic compounds).

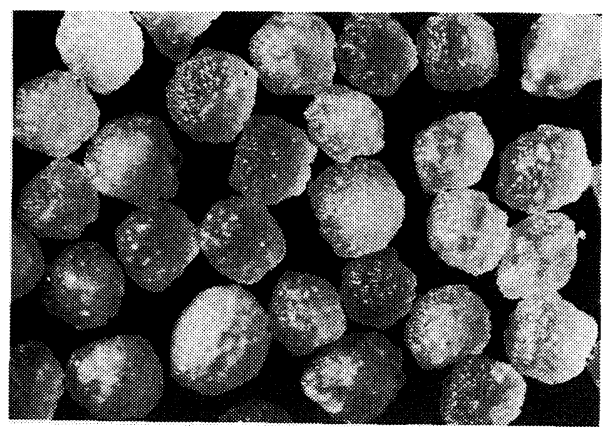

Figure 42. A particular particle form copolymer $(\times 10)$ obtained with the catalyst type $\mathrm{C}$ supported on organic compounds.

catalysts with a well defined shape and particle size, are employed (Figure 41), the elastomers so obtained have equally well defined shape and size (Figure 42).

Polymer J., Vol. 17, No. 1, 1985

\section{CONCLUSIONS}

The most recent, very important development in the field of Z-N catalysis, is the identification of high yield catalysts capable of synthesizing elastomeric products. The new $\mathrm{MgCl}_{2}$ based catalysts are capable of doing a job up to now considered impossible. Furthermore, due to the characteristics of these catalysts, their marrriage with the suspension process is a very convenient goal.

It can be safely stated that the high yield suspension process for ethylene/propylene elastomers is becoming the twin of the PP high yield process already developed by MONTEDISON and presently used by HIMONT.

\section{REFERENCES}

1. P. Galli and L. Noristi, 5th European Plastic Caoutchouc Conference, Vol. 1, A5/1-A5/5, Paris, 1978.

2. P. Galli, L. Luciani, and G. Cecchin, Angew. Makromol. Chem., 94, 63-89 (1981).

3. P. Galli, Abstracts of Papers, IUPAC Symposium, Firenze, Italy, 1980, pp 7-10.

4. P. Galli, Presented at the 28th IUPAC International Symposium on Macromolecules (MACRO 82), July 1982, Amherst, Mass.

5. W. M. Speri and G. R. Patrick, Polym. Eng. Sci., 15, No. 9, 668 (1975).

6. R. C. Thamm, Rubber Chem. Technol., 50, 24 (1977).

7. J. Karger-Kocsis, A. Kalló, A. Szatner, G. Bodor, and Zs. Sényei, Polymer, 20, 37 -43 (1979).

8. F. C. Stehling, T. Huff, C. S. Speed, and G. Wissler, J. Appl. Polym. Sci., 26, 2693 (1981).

9. P. Galli, 5 ème Convegno AIM, Milano, Italy, 1981, $3-20$.

10. T. Simonazzi, A. Savadori, and E. Marchetti, IUPAC International Symposium on Macromolecules, Athens, 1982.

11. P. Galli, T. Simonazzi, and S. Danesi, International Symposium on Macromolecules, Capri, Italy, 1983.

12. C. B. Bucknall, "Toughened Plastics," Applied Science Publishers, Ltd., London, 1977.

13. G. Natta, G. Crespi, A. Valvassori, and G. Sartori, Rubber Chem. Technol., 36, 1583 (1963).

14. G. P. Guidetti, P. Busi, I. Giulanelii, and R. Zannetti, Atti del Convergno su "Applicazioni delle tecniche RX" Bressanone, Italy, Marzo, 1983.

15. T. Simonazzi and L. Abis, Atti del III Convergno AIM, Gargnano, Italy, 1981, 385-399.

16. S. Davison and G. L. Taylor, Br. Polym. J., 4, 65 (1972).

17. N. M. Seidov, F. O. Guseinov, Kh. D. Ibrahimov, A. I. Abasov, and M. A. Efendiyev, Vysokomol. Soyedin. Ser. A, 19, 1523 (1977). 